\title{
The Effect of Concurrent Aerobic and Anaerobic Exercise on Stress, Anxiety, Depressive Symptoms, and Blood Pressure in Renal Transplant Female Patients: A Randomized Control Trial
}

\author{
Elham Shakoor \\ Department of Sport Physiology, School of Physical Education and Sport Sciences, Shiraz University, Shiraz, Iran \\ E-mail: eli_shakoor@yahoo.com \\ Mohsen Salesi (Corresponding author) \\ Department of Sport Physiology, School of Physical Education and Sport Sciences, Shiraz University, Shiraz, Iran \\ E-mail: mhsnsls@gmail.com \\ Maryam Koushki \\ Department of Sport Physiology, School of Physical Education and Sport Sciences, Shiraz University, Shiraz, Iran \\ E-mail: koushkie53@yahoo.com \\ Enayatollah Asadmanesh \\ Department of Sport Physiology, School of Physical Education and Sport Sciences, Shiraz University, Shiraz, Iran \\ E-mail: e_asadmanesh@yahoo.com
}

Darryn S. Willoughby

Department of Health, Human Performance, and Recreation, Exercise and Biochemical Nutrition Laboratory, Baylor University, Waco, TX, USA Email: darryn_willoughby@baylor.edu

Ahmad Qassemian

Department of Sport Physiology, School of Physical Education and Sport Sciences, Shiraz University, Shiraz, Iran Email: ahmadqassemian@gmail.com

Received: 12-09- 2016

Accepted: 20-10- 2016

Published: 31-10- 2016

doi:10.7575/aiac.ijkss.v.4n.4p.25

URL: http://dx.doi.org/10.7575/aiac.ijkss.v.4n.4p.25

\begin{abstract}
Background: Prevalence of stress, anxiety, depressive symptoms, and high blood pressure are known to be important issues among renal transplant patients. Objective: The main purpose of this study was to evaluate the effect of selected exercises on blood pressure, stress, anxiety, depressive symptoms, and blood pressure among renal transplant patients. Method: Thirty two women patients (aged, 20-50 years) with 2 to 3 years post renal transplantation history were voluntarily and objectively recruited. Participants were randomly divided into two groups, exercise ( $\mathrm{n}=16)$ and control $(n=16)$. The exercise group performed 10 weeks of exercise 3 days per week, and for 60-90 minutes per session. The control group involved no exercise. The DASS21 questionnaire was used to collect psychological data, and blood pressure was measured before and after 10 weeks of exercise. Data analysis was conducted using dependent and independent t-tests. Results: Concurrent exercise significantly reduced anxiety, stress, depressive symptoms, and systolic blood pressure in the exercise group only $(\mathrm{p}=0.000)$. Conclusion: Ten weeks of low-intensity exercise can be an effective measure to improve the stress, anxiety, depressive symptoms, and blood pressure in renal transplant patients. Our results suggest that a regular pattern of selected exercises can be effective on stress, anxiety, depressive symptoms, and blood pressure and may be beneficial for renal transplant patients.
\end{abstract}

Keywords: Selected exercise, Stress, Anxiety, Depressive symptoms, Renal transplant

\section{Introduction}

End-stage renal failure is an irreversible progressive renal dysfunction in which the body's ability to maintain fluid and electrolyte balance is lost, leading to uremia or azotemia (Rigatto et al. 2000). The number of people suffering from end-stage renal disease is increased by 6\% annually, and 25,000 patients have been reported in Iran (Mahdavi-Mazdeh 
2012). End-stage chronic renal failure patients cannot survive without replacement therapy (Rigatto et al. 2000). In Iran, $52.7 \%$ of patients undergo hemodialysis (Kargarfard et al., 2015) and $45.5 \%$ benefit from renal transplantation (Mousavi, Soleimani, and Mousavi 2014). With regard to problems like dialysis machine dependence, anxiety and high cost patients often prefer to undergo renal transplantation to survive (Painter et al. 2002; Sorensen et al. 2012; Boostani and Ghorbani 2014). Renal transplantation is currently an effective method in the treatment of advanced chronic renal failure (Spitzer and Avner 2012). Despite the benefits of renal transplantation, patients are confronted with a host of new problems after transplantation (Boostani and Ghorbani 2014).

Despite the benefits of kidney transplantation, it is associated with specific complications and can lead to social and psychological problems (Masoudi Alavi, Sharifi, and Aliakbarzadeh 2009). The prevalence of psychiatric illnesses before and two months after transplantation has been reported to be $11.1 \%$ and $36.1 \%$, respectively. Depression and anxiety are reported to be common among patients with renal failure (Masoudi Alavi, Sharifi, and Aliakbarzadeh 2009). In the study of Dobbels et al (2008) concerning depression in renal transplant recipients, which was performed on 47,899 patients, 3,360 patients were depressed after 3 years (Dobbels et al. 2008). In a study conducted in Tehran, the rate of depression in patients with renal transplantation was reported similar to dialysis patients (Masoudi Alavi, Sharifi, and Aliakbarzadeh 2009). Hypertension is among the health problems (Kargarfard et al., 2016) of renal transplant patients, which is a common complication after renal transplantation (Kasiske et al. 2000). The prevalence of hypertension after renal transplantation in combination with cyclosporine is 60-80\% (Magee and Milford 2004). In another study, the prevalence of hypertension has been reported to be $90 \%$ after renal transplantation (Budde et al. 1997). Recent studies have indicated that lifestyle can be effective in development of stress, anxiety and psychological pressures, which influence hypertension (Sadeghi et al., 2016; Sadeghi, Shariat, Asadmanesh, \& Mosavat, 2013).

There is a clear relationship between sport and leisure activity with resilience and stress (Ma et al. 2013). A recent study (Pooranfar et al. 2014) found that 10 weeks of exercise training improved the quality and quantity of sleep, as well as a number of sleep-related physiological parameters (Shakoor, koushki Jahromi, \& Sadeghi, 2015; Shariat, Kargarfard, \& Sharifi, 2012) in renal transplant recipients, and would be an effective approach to treat sleep-related disorders (Shariat et al., 2015)in renal transplant recipients. Another study found significant beneficial effects of regular exercise on physical fitness, walking capacity, cardiovascular dimensions (e.g. blood pressure and heart rate), health-related quality of life (Afzalpour et al., 2016), and some nutritional parameters in adults with chronic kidney disease (Heiwe and Jacobson 2011). On the other hand, there is considerable evidence of numerous beneficial effects of physical activity on the health of patients with renal disease (Johansen 2005), which can reduce symptoms of anxiety and depressive symptoms, and promoting mood and feelings of well-being (Mazzoni et al. 2014). Given the above facts, the objective of present study was to evaluate the impact of a 10-week concurrent aerobic and anaerobic exercise program on stress, anxiety, depressive symptoms and hypertension in renal transplant patients.

\section{Methods}

\subsection{Study design and participation}

This study utilized a randomized control trial research design. The current study involved an experimental pretestposttest design with test and control groups. Fifty women patients admitted to Nemazee Hospital in Shiraz, Iran with age range of 20 to 50 years satisfying the conditions of entry into the study (2 to 3 years after renal transplantation) were selected based on targeted purposive sampling, and were randomly divided into exercise $(n=17)$ and control $(n=$ 17) groups. After selection, all participants read and signed an informed consent form. All participants then completed a questionnaire before examination by a physician to check the medical condition and exclusion criteria. All participations did not consume dietary supplements (e.g. proteins, carbohydrates, amino acids) at least three months before this study (Shariat et al. 2015). The exclusion criteria included cardiovascular problems and chronic diseases (e.g. heart disease, stroke, diabetes, cancer) The inclusion criteria included the ability to walking and low-intensity running on treadmill, and the ability to pedal on cycle ergometer (Ulubay et al. 2006). The participants were not allowed to participate in other physical activity exercises during the study, and both groups were advised to continue with a normal sleep pattern of approximately 8 hours per night for the duration of the experimental study period. This study was in agreement with the principle of Helsinki Declaration and approved by the ethical committee in the Shiraz Medical University, in Iran. Finally, two participants (one in exercise and one in control group) did not finish the intervention due to personal reasons. Therefore, the final sample was 32 females, 16 in the exercise group and 16 in the control group. The progress through the phases of screening, enrolment, allocation, post-testing, and data analysis is illustrated in Figure 1. 


\section{Enrollment}

Assessed for eligibility $(n=40)$

Excluded $(\mathrm{n}=8$ )

Not meeting inclusion criteria $(\mathrm{n}=6)$

Declined to participate $(\mathrm{n}=1)$

Other reasons $(\mathrm{n}=1)$

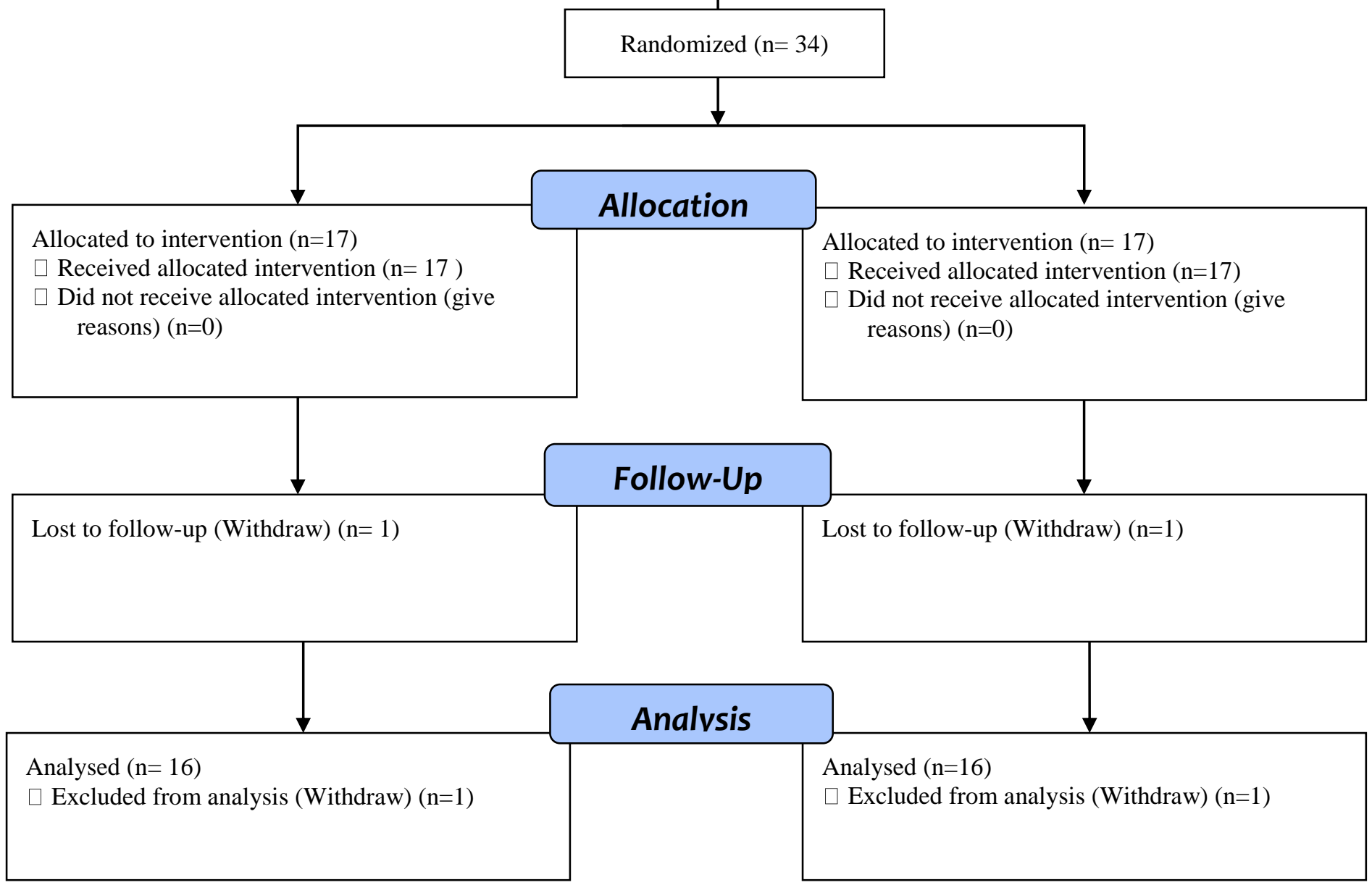

Figure 1. Flow diagram of the progress through the phases of a parallel randomized trial

\subsection{The exercise protocol}

The test group participants participated in an exercise program for ten weeks. The exercise program was designed by an experienced coach of conditioning under supervision of a certified strength and conditioning specialist (CSCS) with regard to the physical condition of patients in terms of the type, intensity, frequency, and repetition of the exercise after a pilot study verifying the safety, efficiency and simplicity (Gordon et al. 2005; Shariat et al. 2015). The experimental group performed the exercise program for 10 weeks, three sessions per week for 60-90 minutes. Each session included a 15-minute warm-up, 20 minutes of aerobic exercise, 20 minutes of resistance exercise, and a 10-minute cool-down including running with a slow pace followed by stretching and light exercises (Johansen 2005). The control group did not participate in any exercise during the 10-week period. Before and after the exercise protocol Research variables (depressive symptoms, anxiety and stress, and plod pressure) were measured.

The participants performed the main workout for 35-55 minutes between 10 to 12 am in a circuit consisting of 9-17 stations, with 3-6 circles per session, 1-2 minutes of rest between each station, and 3-5 minutes of rest between circles (Pooranfar et al. 2014; Painter et al. 2003) which was a combination of aerobic exercises using a bicycle ergometer (Nautilus, Vancouver, WA, USA), treadmill (Nautilus, Vancouver, WA, USA) and resistance exercises with free weights (Iron Grip Barbell Company, Santa Ana, CA, USA). The aerobic exercise was done on a stationary bicycle or treadmill with 40-70\% maximum heart rate and the resistance exercise involved 45- 65\% intensity of 1 maximum repetition (1RM). The aerobic exercise was performed with mild- to moderate-intensity corresponding to $40-60 \%$ maximum VO2. After 10 minutes of general warm-up exercises and stretching, the resistance exercise group participants started the testing session. The aerobic exercises were performed for 30 minutes with an increase of 10 minutes in duration every 4 weeks during 10 weeks (Baria et al. 2014; Shakoor et al. 2015). 


\subsection{Data collection procedure}

On the first day of the sampling process, blood pressures of the participants were measured by a sport physiologist after 5 minutes in the sitting position. Blood pressures were also taken 30 minutes before exercise and after 10 weeks of exercise training. The DASS-21 questionnaire (depressive symptoms, anxiety and stress scale (DASS-21), a valid and reliable questionnaire to measure depressive symptoms (Johansen 2005), was completed by each participants, under the supervision of researcher before the exercise protocol and after 10 weeks of exercise. DASS21 is a self-test questionnaire for depressive symptoms, anxiety and stress. It consists of 21 questions in three equal parts ( 7 items each) concerning each of the indexes under consideration (Salehi Fadardi 2009).

\subsection{Statistical analysis}

To evaluate the changes in Stress, Anxiety, Depressive symptoms and blood pressure (Systolic and Diastolic) across the experiments, a two way repeated-measure ANOVA with a within-participants factor (pre and post-test) and a betweenparticipants factor (experiment and control) was performed. Analysis of data was performed with the Statistical Package for Social Scientists (SPSS) version 20 (IBM, New York, USA). The Levene test was employed to check sphericity, and normal distribution was verified using the Kolmogorov-Smirnov adaptation test. An $\alpha$-level of $<0.05$ was accepted as statistically significant.

\section{Results}

The distribution of dependent variables in both groups was subjected to normality testing. Table 1 shows the result of within participants and the interaction. According to the results for all research variables, there were significant differences between pre- and post-test for all variables.

Table1. Result of ANOVA within - between participants effects

\begin{tabular}{|c|c|c|c|c|c|}
\hline Variable & Source & MS & $\mathbf{F}$ & p value & $\eta 2$ \\
\hline \multirow[t]{2}{*}{ Stress } & test & 141.016 & 25.528 & $<0.001$ & 0.460 \\
\hline & test $*$ group & 165.766 & 30.008 & $<0.001$ & 0.500 \\
\hline \multirow[t]{2}{*}{ Anxiety } & test & 107.641 & 44.104 & $<0.001$ & 0.595 \\
\hline & test $*$ group & 92.641 & 37.958 & $<0.001$ & 0.559 \\
\hline \multirow[t]{2}{*}{ Depression } & test & 112.891 & 39.8 & $<0.001$ & 0.570 \\
\hline & test $*$ group & 102.516 & 36.142 & $<0.001$ & 0.546 \\
\hline \multirow[t]{2}{*}{ Systolic } & test & 189.063 & 3.017 & 0.093 & 0.091 \\
\hline & test $*$ group & 1806.25 & 28.828 & $<0.001$ & 0.490 \\
\hline \multirow[t]{2}{*}{ Diastolic } & test & 19.141 & 0.406 & 0.529 & 0.013 \\
\hline & test $*$ group & 478.516 & 10.146 & 0.003 & 0.253 \\
\hline
\end{tabular}

According to the result of analysis variance, the interaction effect was significant for all variables hence mean comparisons between pre and post-test was performed for all variables separately (Table 2). The results revealed a significant difference between pre and post-test for the exercise group only, while there was no significant difference between pre and post-test for the control group.

Table 2. Bonferroni post hoc test for comparing between and within groups

\begin{tabular}{|c|c|c|c|c|c|c|}
\hline & & Pre test & $\begin{array}{c}\text { between } \\
\text { groups } \\
\text { diff** }\end{array}$ & Post test & $\begin{array}{l}\text { between } \\
\text { groups } \\
\text { diff }\end{array}$ & $\begin{array}{c}\text { within groups } \\
\text { diff* }\end{array}$ \\
\hline Variable & Groups & & P value & & P value & $P$ value \\
\hline \multirow[t]{2}{*}{ Systolic } & exercise & $124.4 \pm 9.1$ & \multirow{2}{*}{0.463} & $110.3 \pm 9$ & \multirow{2}{*}{$<0.001$} & $<0.001$ \\
\hline & control & $126.6 \pm 7.5$ & & $133.8 \pm 7.4$ & & .055 \\
\hline \multirow[t]{2}{*}{ Diastolic } & exercise & $75.9 \pm 11.7$ & \multirow{2}{*}{0.271} & $71.6 \pm 7.9$ & \multirow{2}{*}{$<0.001$} & .082 \\
\hline & control & $80.3 \pm 7.4$ & & $86.9 \pm 7.7$ & & .091 \\
\hline \multirow[t]{2}{*}{ Stress } & exercise & $11.7 \pm 3.6$ & \multirow{2}{*}{0.262} & $5.5 \pm 2.9$ & \multirow{2}{*}{0.001} & $<0.001$ \\
\hline & control & $10.1 \pm 4.1$ & & $10.4 \pm 4.3$ & & 0.766 \\
\hline \multirow[t]{2}{*}{ Anxiety } & exercise & $7.5 \pm 3.7$ & \multirow{2}{*}{0.607} & $2.5 \pm 2.6$ & \multirow{2}{*}{0.009} & $<0.001$ \\
\hline & control & $6.7 \pm 5$ & & $6.5 \pm 5.1$ & & 0.737 \\
\hline \multirow[t]{2}{*}{ Depression } & exercise & $8.9 \pm 3.8$ & \multirow{2}{*}{0.391} & $3.8 \pm 2.4$ & \multirow{2}{*}{0.009} & $<0.001$ \\
\hline & control & $7.6 \pm 4.7$ & & $7.5 \pm 4.8$ & & 0.835 \\
\hline
\end{tabular}




\section{Discussion}

The results showed that a round of physical activity including aerobic and anaerobic exercise can reduce systolic blood pressure, stress, anxiety and depressive symptoms in renal transplant patients. One goal of this study was to evaluate the effect of exercise on blood pressure in renal transplant patients. As these patients are usually overweight and have high blood pressure, exercise can be useful to reduce their blood pressure. The results of this study showed that performing 10 weeks of concurrent exercise has a significant effect in reduction of systolic blood pressure. However, with respect to the studies on other participants, the results of our study were consistent with the following studies. Christen and Johansson (Johansen 2005) reported reduced blood pressure after an aerobic exercise in patients with chronic renal failure. In addition, physical activity reduces systolic blood pressure in patients with hypertension. However, Miller et al (Miller et al. 2002) found no significant difference in blood pressure after 6 months of physical activity in hemodialysis patients. The difference between our study and the mentioned studies can be probably attributed to the difference in the intensity and duration of exercise programs as well as the difference between age and sex of participants, the preparation level of participants and their health status. The aerobic exercise programs significantly reduce blood pressure of the kidney patients. some clinical advantage of aerobic and anaerobic exercise has been wellknown in hypertensive and normotensive individuals (Nybo et al. 2010) as well as in CKD patients (Boyce et al. 1997). Aerobic exercise lowers blood pressure by reducing peripheral vascular resistance due to the improvement of endothelium-mediated vasodilatation, attenuation of increased sympathetic nervous system activity and vascular remodeling (Baria et al. 2014).

The exact mechanism of the effect of exercise on blood pressure reduction is unknown; although, it may be attributed to catecholamines produced by the exercise. This reaction is involved in reduced peripheral resistance to blood flow and subsequent reduction of blood pressure (Painter et al. 2003; Pooranfar et al. 2014). Physical activity can facilitate the excretion of sodium by the kidneys, resulting in reduced fluid volume and blood pressure. It seems that exercise can reduce blood pressure by increasing the number of capillaries in active skeletal muscle, increased cardiac output, reduced vascular resistance due to dilatation, decreased resistance to blood flow, improved neural regulation of blood vessels, reduced peripheral resistance, decreased heart rate during rest and activity and changes of body weight (Baria et al. 2014). Such adaptations increase the transverse cavity area and result in improved venous dilatation, such that increased blood flow during exercise can trigger the waste removal process, which can be effective in improvement and control of blood pressure (Shinn et al. 2001). In the present study, after data collection and statistical analysis, the results indicated that the level of stress, anxiety and depressive symptoms was lower in renal transplant patients of test group performing the designed exercised program during 10 weeks relative to control group. The results of our study were in line with previous studies (Painter et al. 2002; Heiwe and Jacobson 2011) which asserted that, although exercise plays a positive role in mental health, it is not effective upon anxiety, stress and depressive symptoms. Meanwhile, Hale (1997) reported that physical outdoor activities have not been accepted as an effective intervention strategy by clinical psychologists and psychiatrists. It seems that the differences between results of this research with other studies are related to factors such as the use of different protocols with various variables and exercise intensities, different physical and exercise conditions of participants, nutritional status, mental-emotional status, sex and age. Exercise reduces neural pressures and depressive symptoms in the work ambient. This phenomenon is explained by increased level of serotonin and norepinephrine during exercise activities, which result in depressive symptoms reduction (Smith and Elliott 2003). In other words, physical training affects human spirit in two ways: endorphin release and reduction of cortisol levels (the hormone secreted in blood after stress). According to physiology specialists, endorphins are natural sedative drugs causing pleasant feelings. Physical training increases the level of endorphin secretion. Some researchers concluded that physical training has a considerable effect in increasing the serotonin level (the hormone Effective in upgrading the mood) (Dunn et al. 2005). Thus, exercise seems to cause increased delivery of endorphin and serotonin to body and preservation of it for a longer period during exercise (Anderson and Shivakumar 2013). Higher consumption of antihypertensive drugs and the incidence of side effects of these medications, as well as weakening of the individuals due to gradual complications of such drugs, can be likely reasons for higher prevalence of depressive symptoms over time. the relative frequency of depression in patients treated with antihypertensive drugs was three times higher than those without high blood pressure, and patients suffering from depression are less likely to cooperate with respect to consumption of antihypertensive drugs (Johansen et al. 2012). A number of other studies support the link between depressive symptoms and high blood pressure (Johansen et al. 2012; Marmot 1985). In one study, evaluation of 508 patients over four years showed no correlation between depressive symptoms and hypertension (Shinn et al. 2001). In another study, a 20-year follow-up study showed that patients with symptoms of depression are afflicted with hypertension much more frequently than those without depression (Jonas and Lando 2000). These contradictory findings indicated that the relationship between depression and hypertension can be a bilateral multifactorial relationship, in which a number of factors play the role of predisposing factors and others the supportive role. Recognizing these factors, with the aim of intervention programs, demands further studies with strong methodologies.

\section{Conclusion}

The present study was designed to determine the effect of 10 weeks concurrent aerobic and anaerobic exercise program can decrease on psychological factor such as stress, anxiety and depressive symptoms reduce systolic blood pressure, in renal transplant patients. The study has gone some way towards enhancing our understanding of exercise program for 
especial participants or patients. A limitation of this study is that the numbers of patients and controls were relatively small. In addition this study was conducted on female and further research needs to examine.

\section{References}

Afzalpour, M. E., Bashafaat, H., Shariat, A., Sadeghi, H., Shaw, I., Dashtiyan, A. A., \& Shaw, B. S. (2016). Plasma protein carbonyl responses to anaerobic exercise in female cyclists. International Journal of Applied Exercise Physiology, 5(1), 53-58.

Anderson, E., and Geetha,S,. (2013). Effects of exercise and physical activity on anxiety. Frontiers in psychiatry 4 (27):1-4.

Baria, F., Maria A., Danilo T.A., Adriano, A., Mariana, L.R, Marco Túlio,M, and Lilian, C. (2014). Randomized controlled trial to evaluate the impact of aerobic exercise on visceral fat in overweight chronic kidney disease patients. Nephrology Dialysis Transplantation 29 (4):857-864.

Boostani, H., and Ali, A. (2014). The comparison of general health status between hemodialysis and kidney transplant patients in university hospitals of Ahvaz, Iran. Journal of Renal Injury Prevention 3 (1):27.

Boyce, M.L, Robert A.R., Pratap S.A., Carlos,R., Angelique, F., Paul.,F, Dan, S., and Chris, N. (1997). Exercise training by individuals with predialysis renal failure: cardiorespiratory endurance, hypertension, and renal function. American Journal of Kidney Diseases 30 (2):180-192.

Budde, K, J Waiser, L Fritsche, J Zitzmann, M Schreiber, R Kunz, and H-H Neumayer. (1997). Hypertension in patients after renal transplantation. Paper read at Transplantation Proceedings.

Dobbels, F, Melissa A.S, Jon J.S, Anne V.T, J Ross,M., and Bertram, L.K. (2008). Depressive disorder in renal transplantation: an analysis of Medicare claims. American Journal of Kidney Diseases 51 (5):819-828.

Dunn, A.L., Madhukar H.T., James B.K, Camillia G, C, and Heather, H. (2005). Exercise treatment for depression: efficacy and dose response. American journal of preventive medicine 28 (1):1-8.

Gordon, E.J, Thomas. P, Laura A.S, Peter J.M, and Ashwini R.S. (2005). Needed: tailored exercise regimens for kidney transplant recipients. American journal of kidney diseases: the official journal of the National Kidney Foundation 45 (4):769.

Heiwe, S, and Stefan H.J. (2011). Exercise training for adults with chronic kidney disease. The Cochrane Library.

Johansen, A, Jostein H, Robert S, and Ottar B. (2012). Anxiety and depression symptoms in arterial hypertension: the influence of antihypertensive treatment. The HUNT study, Norway. European journal of epidemiology 27 (1):63-72.

Johansen, K.L. (2005). Exercise and chronic kidney disease. Sports Medicine 35 (6):485-499.

Jonas, B. S, and James F.L. (2000). Negative affect as a prospective risk factor for hypertension. Psychosomatic Medicine 62 (2):188-196.

Kargarfard, M., Lam, E. T. C., Shariat, A., Shaw, I., Shaw, B. S., \& Tamrin, S. B. M. (2016). Efficacy of massage on muscle soreness, perceived recovery, physiological restoration and physical performance in male bodybuilders. Journal of Sports Sciences, 34(10), 959-965.

Kargarfard, M., Shariat, A., Shaw, B. S., Shaw, I., Lam, E. T. C., Kheiri, A., .. Tamrin, S. B. M. (2015). Effects of Polluted Air on Cardiovascular and Hematological Parameters After Progressive Maximal Aerobic Exercise. Lung, 193(2), 275-281.

Kasiske,.B.L, Miguel A, William E, Robert S, Gabriel M, Robert S, David R,. (2000). Recommendations for the outpatient surveillance of renal transplant recipients. Journal of the American Society of Nephrology 11 (suppl 1):S1S86.

Ma, L, Hong, L, Yueh-Min, L, Hsiang-Li, H, Lan, L, Mei-Yu, L, and Kuo-Cheng, L. (2013). The relationship between health-promoting behaviors and resilience in patients with chronic kidney disease. The Scientific World Journal, 12(3), 76-79.

Magee, C, and Edie, M. (2004). Clinical aspects of renal transplantation. Brenner BM. Brenner \& Rector's The kidney. 7th ed. Philadelphia: WB. Saunders, Elsevier:2810-6.

Mahdavi-Mazdeh, M. (2012). The Iranian model of living renal transplantation. Kidney international 82 (6):627-634.

Marmot, M.G. 1985. Psychosocial factors and blood pressure. Preventive medicine 14 (4):451-465.

Masoudi, A, Khadije, Sh, and Zahra, A. (2009). Depression and anxiety in patients undertaken renal replacement therapy in Kashan during 2008. KAUMS Journal (FEYZ) 12 (4):46-51.

Mazzoni, D, E Cicognani, G Mosconi, V Totti, GS Roi, M Trerotola, and A Nanni Costa. (2014). Sport Activity and Health-Related Quality of Life After Kidney Transplantation. Paper read at Transplantation Proceedings.

Miller, B.W, Cheryl,L, Mary, E, Darlene, H, and Mark, A. (2002). Exercise during hemodialysis decreases the use of antihypertensive medications. American Journal of Kidney Diseases 39 (4):828-833. 
Mousavi, S, Soleimani, A and Mousavi,M. 2014. Epidemiology of end-stage renal disease in Iran: A review article. Saudi Journal of Kidney Diseases and Transplantation 25 (3):697.

Nybo, L, Emil, S, Markus, D, Magni,M, Therese,H, Lene,S, Jens,B, Morten,B, Jens,J, and Per,A. (2010). High-intensity training versus traditional exercise interventions for promoting health. Med Sci Sports Exerc 42 (10):1951-8.

Painter, L, Lisa,H, Karen,R, Liliana,L, Steven,M, Marylin,D, Stephen,L, and Nancy,L. 2003. Effects of exercise training on coronary heart disease risk factors in renal transplant recipients. American journal of kidney diseases 42 (2):362-369.

Painter, P, Lisa,H, Karen,R, Liliana,L, Suzanne,D, Steven,P, Stephen,T, and Nancy,L. (2002). A randomized trial of exercise training after renal transplantation. Transplantation 74 (1):42-48.

Pooranfar, S, E Shakoor, MJ Shafahi, M Salesi, MH Karimi, J Roozbeh, and M Hasheminasab. (2014). The Effect of Exercise Training on Quality and Quantity of Sleep and Lipid Profile in Renal Transplant Patients: A Randomized Clinical Trial. International journal of organ transplantation medicine 5 (4):157.

Rigatto, C, Robert,N, Gloria,M, Ronald, G, and Patrick,S. 2000. Long-term changes in left ventricular hypertrophy after renal transplantation. Transplantation 70 (4):570-575.

Sadeghi, H., Hakim, M. N., Hamid, T. A., Amri, S. Bin, Razeghi, M., Farazdaghi, M., \& Shakoor, E. (2016). The Effect of Exergaming on Knee Proprioception in Older Men: A Randomized Controlled Trial. Archives of Gerontology and Geriatrics.

Sadeghi, H., Shariat, A., Asadmanesh, E., \& Mosavat, M. (2013). The Effects of Core Stability Exercise on the Dynamic Balance of Volleyball Players. International Journal of Applied Exercise Physiology, 2(2), 1-10.

Salehi,F, J. 2009. A comparative study of anxiety, stress, and depression in physically abused and non-abused Iranian wives. Iranian Journal of Psychiatry and Behavioral Sciences 3.

Shakoor, E, Maryam,K., Mohsen,S, and Hassan, S. 2015. The Effects of 10 Weeks Concurrent Aerobic and Strength Exercise on Quality of Life and Resilience of Kidney Transplant Patients. International Journal of Applied Exercise Physiology 4 (2):1-8.

Shariat, A., Bahri Mohd Tamrin, S., Daneshjoo, A., \& Sadeghi, H. (2015). The Adverse Health Effects of Shift Work in Relation to Risk of Illness/Disease: A Review. Acta Medica Bulgarica, 42(1), 63-72.

Shariat, A., Kargarfard, M., \& Sharifi, G. R. (2012). The effect of heavy resistance exercise on circadian rhythm of salivary cortisol in male body building athletes. Journal of Isfahan Medical SchooL (I.U.M.S), 29, 2400-2412.

Shariat, A, Kargarfard,M., Danaee, M., and Tamrin, SBM. (2015). Intensive resistance exercise and circadian salivary testosterone concentrations among young male recreational lifters. The Journal of Strength \& Conditioning Research 29 (1):151-158.

Shinn, E., Walker,S., Kay, T., Sachik, T, and John, P. (2001). Blood pressure and symptoms of depression and anxiety: a prospective study*. American Journal of Hypertension, 14 (7), 660-664.

Smith, LL, and CH Elliott. 2003. Demystifying and defeating depression. Depression for dummies. New Jersey, NJ: Wiley.

Sorensen, E, Mark,J, Hocine, T., Tammy, S., Lena,M., Bethany,K., Kristina,L., and Daniel,E,. (2012). The kidney disease quality of life cognitive function subscale and cognitive performance in maintenance hemodialysis patients. American Journal of Kidney Diseases, 60 (3), 417-426.

Spitzer, A, and Ellis,D. (2012). Inheritance of kidney and urinary tract diseases. Vol. 9: Springer Science \& Business Media.

Ulubay, G., B. Akman, S. Sezer, K. Calik, F. Eyuboglu Oner, N. Ozdemir, and M. Haberal. (2006). Factors Affecting Exercise Capacity in Renal Transplantation Candidates on Continuous Ambulatory Peritoneal Dialysis Therapy. Transplantation Proceedings, 38 (2), 401-405. 